\title{
High voltage electric double layer capacitor using a novel solid-state polymer electrolyte
}

\author{
Full length articles
}

\section{Takaya Sato $^{1,3 *}$, Shoko Marukane ${ }^{1}$, Takashi Morinaga ${ }^{1}$, Toshio Kamijo ${ }^{1}$, Hiroyuki Arafune ${ }^{1}$, Yoshinobu Tsujii ${ }^{2,3}$}

${ }^{1}$ National Institute of Technology, Tsuruoka College, Department of Creative Engineering, 104 Sawada, Inooka, Tsuruoka, Yamagata 997-8511, Japan

${ }^{2}$ Institute for Chemical Research, Kyoto University, Gokasho, Uji, Kyoto 611-0011, Japan

${ }^{3}$ Core Research for Evolutional Science and Technology (CREST), Japan Science and Technology Agency, Tokyo 102-0076, Japan

${ }^{*}$ Corresponding author.

Tel \& Fax: +81-23-525-9461

E-mail: takayasa@tsuruoka-nct.ac.jp 


\section{Abstract}

We designed and fabricated a bipolar-type electric double layer capacitor (EDLC) with a maximum $7.5 \mathrm{~V}$ operating voltage using a new concept in solid electrolytes. A cell having a high operating voltage, that is free from liquid leakage and is non-flammable is achieved by a bipolar design utilizing a solid polymer electrolyte made up of particles in a three-dimensional array, such as crystals composed of $75 \mathrm{wt} \%$ of hybrid particles decorated with a concentrated ionic liquid polymer brush (PSiP) and $25 \mathrm{wt} \%$ of an ionic liquid (IL). The resulting solid film had sufficient physical strength and a high enough ionic conductivity to function as an electrolyte. Solidification as well as ionic conduction is due to the regular array of PSiPs, thereby producing a high ion-conductivity from a networked path between cores containing an appropriate amount of IL as a plasticizer. The demonstration cell shows a relatively good cycle durability and rate properties up to a $10 \mathrm{C}$ discharge process. It also has a very small leakage current in continuous charging and better self-discharge properties, even at $60{ }^{\circ} \mathrm{C}$, compared with conventional cells. This paper demonstrates the first successful fabrication of a bipolar EDLC in a simple structure using this novel polymer solid electrolyte.

Keywords: Electric double layer capacitor; ionic liquid; polymer solid electrolyte; bipolar cell design; high voltage. 


\section{Introduction}

An electric double layer capacitor (EDLC) is an electrical energy storage device that physically stores electricity by forming an electric double layer consisting of adsorbed layers of cations and anions at the interfaces between the electrodes and the electrolyte. Both the charge and discharge processes of this type of device are nonchemical reactions. Rather, they are Faradaic reactions, unlike a secondary battery and therefore it has a higher rate characteristic can be charged and discharged at a large current, and has a very long cycle life compared with rechargeable batteries [1-3]. Such devices are considered to be promising as a power source for mobile objects such as an automated guided vehicle, a hybrid electric vehicle and construction equipment. In these applications in particular, the high output performance of the EDLC are preferable for use in assisting the acceleration and smooth recovery of regenerative energy during deceleration.

Power density is an index of energy release and recovery of speed to/from the load. The maximum power $\left(P_{\max }\right)$ of EDLCs is represented by the following equations:

$$
P_{\text {max }}=\mathrm{V}_{0}^{2} / 4 \mathrm{R}
$$

Here, $\mathrm{V}_{0}$ and $\mathrm{R}$ are the fully charged voltage of the EDLC and the internal resistance of the cell calculated from the voltage drop (IR drop) at the starting point of the discharge, respectively. The power density is defined either per weight $(\mathrm{W} / \mathrm{kg})$ or per volume $(\mathrm{W} / \mathrm{L})[4]$. Increasing the cell voltage of an EDLC is a very important development goal, because the power density is proportional to the 
square of the cell voltage. Over the last ten years, researchers and developers have succeeded in improving the power and the energy density of EDLCs by using ionic liquids (ILs) as electrolyte solutes [5-7]. Generally, a salt having a melting point lower than $100{ }^{\circ} \mathrm{C}$ is defined as an ionic liquid. Ionic liquids are nonvolatile, have flame retardant properties and a relatively high ionic conductivity [8]. One manufacturer in Japan developed a high performance EDLC with a 3V working voltage using an electrolyte that had a quaternary ammonium ionic liquid diluted with propylene carbonate. Some ionic liquids have a higher resistance to electrochemical degradation in the EDLC cell and a have a higher solubility in organic solvents used as an electrolyte than the solid ammonium salts conventionally used for EDLC electrolytes. Hence, using ILs, it is possible to make an electrolyte with high conductivity and durability against electrochemical degradation, resulting in a high power EDLC [3].

On the other hand, the most common cause of failure of electrochemical device products using liquid electrolytes is said to be liquid leaks. For the purposes of weight and cost reduction for the device, aluminum laminate film is used as the packaging material for many devices. However, because the solute in the electrolyte has surfactant properties, the electrolyte may continue to gradually penetrate the heat sealing portion of the package, and so it is very difficult to eliminate the possibility of leakage. With the aim of leakage prevention, much research on solid and gel electrolyte research has been performed to provide a large surface area with thin prismatic cell design and high processability [9-14]. As 
the electrochemical device involves movement of ions in the cell, a decrease in ionic mobility by solidification of electrolyte leads to spoiling of the power performance of the devices. Thus, a gel electrolyte with high ionic mobility is preferable and the fundamental concept of gel-polymer electro-chemical devices is to solidify the electrolyte by using the smallest possible amount of polymer material as a guest for the electrolyte [15]. There are some reports that an EDLC using a gel electrolyte can achieve higher capacity and power compared to a liquid system by making intimate contact between the active material on the electrode and the gel electrolyte [12-14].

Amid such developments, we have developed a totally new concept for the fabrication of a non-volatile, liquid leakage free and non-flammable solid electrolyte having a high ion-conductivity channel in a continuous network. This was constructed for the first time by three-dimensional self-assembly in crystal like structure of hybrid silica particles (PSiPs) having "concentrated" polymer brushes (CPBs) of ionic-liquid type polymers on the silica surface and a small amount of ionic liquid (IL) that is included as a plasticizer. The regular array of CPB-modified particles was expected to provide a polymer-brush realm that was three-dimensionally continuously connected as a network array, in which the chain ends of graft polymers would be concentrated near the boundary region, leading to the expectatation of enhancing the ion conduction in association with the segmental motion (Fig. 1) [16]. Since this polymer electrolyte, an ionic liquid type polymer-brush decorated silica particles / İonic liquids composite solid electrolyte 
(PSiP/IL solid electrolyte), also had good mechanical strength and a good ability to retain liquids, it became possible to produce a bipolar design that can provide a high voltage EDLC by a series connection between the electrode units in the cell package. Here we will present the design and the electrochemical performance of a high working voltage (7.5V) EDLC cell using a PSiP/IL solid electrolyte.

<Fig. 1>

\section{Experimental}

\subsection{Materials}

To prepare the EDLC electrolytes, the ionic liquid, $N, N$-diethyl- $N$-(2-methoxyethyl)- $N$-methylammonium $\quad$ bis(trifluoromethylsulfonyl)imide $\quad$ (DEME-TFSI) was purchased from Kanto Chemical Company Inc. and it was used after dehydration with molecular sieve (4A). The silica particles decorated with concentrated ionic liquid polymer brushes ( $\mathrm{PSiP}$ ) was prepared by the procedures described in our previous paper [16]. The preparation process is summarized in the schematic figure Fig. 1. We performed a surface-initiated atom transfer radical polymerization (ATRP) - a type of living radical polymerization (LRP) - by using $N, N$-diethyl- $N$-(2-methacryloylethyl)- $N$-methylammonium bis(trifluoromethylsulfonyl)imide (DEMM-TFSI), a polymerizable ionic liquid as a monomer. This polymerization was started from the ATRP-initiating groups on the surface of monodisperse, $130 \mathrm{~nm}$ diameter, silica particles (SiP). This ATRP was well 
controlled, and gave a hybrid silica particle (PSiP) with an IL type of CPB with a relatively low polydispersity index and molecular weights up to about 100,000. The PSiP used for the solid electrolyte was obtained by polymerization at $70^{\circ} \mathrm{C}$ for $14 \mathrm{~h}$ giving a polymer of $M n=25,000$ and polydispersity index $(M w / M n)$ of 1.13 at a monomer conversion of $52 \%$, where $M n$ and $M w$ are the number and weight-average molecular weight, respectively. The graft density per unit area was estimated to be 0.15 chains $\mathrm{nm}^{-2}$. The surface occupancy (dimensionless graft density), $\sigma *$ was 0.33 , indicating that $33 \%$ of the silica surface is occupied by the cross-sectional area of the fully stretched polymer brushes; for the calculation, the bulk density of poly(DEMM-TFSI) was assumed to be $1.4 \mathrm{~g} \mathrm{~cm}^{-3}$. From the $\sigma *$ value, the ionic-polymer brush on the PSiP for the solid electrolyte was reasonably categorized as being in the CPB regime [16].

\subsection{Preparation of electric double layer capacitor cell}

The following procedure, illustrated in Fig. 2, was used for the fabrication of the high voltage EDLC cell. The carbon paint, comprising carbonized palm shell charcoal (surface area $2000 \mathrm{~m}^{2} \mathrm{~g}^{-1}$, average pore diameter $20 \mathrm{~nm}$, average particle size $8 \mu \mathrm{m}$ ), acetylene black and poly(vinylidene fluoride) [PVDF; average $M \mathrm{w}$ ca. 534,000, purchased from Sigma-Aldrich Fine Chemicals Co. Ltd.] in $N$-methyl-2-pyrrolidone (NMP), was coated by a screen printing method using a HP-320 hand printer (Newlong Seimitsukogyo Co. Ltd.) with a thin metal mask (Bon Mark Co. Ltd.) onto one side of an aluminum oxide foil (30 $\mu \mathrm{m}$ thick). The 
active electrode layer was dried at $80{ }^{\circ} \mathrm{C}$ for $1 \mathrm{~h}$ in an oven to remove NMP and moisture. Subsequently, the dried electrode was pressed in a roll press at 50MPa and further dried at $100{ }^{\circ} \mathrm{C}$ in vacuum for $15 \mathrm{~h}$. The electrode obtained in this way was composed of $90 \mathrm{wt} . \%$ palm shell charcoal, $5 \mathrm{wt} . \%$ acetylene black and $5 \mathrm{wt} . \%$ PVDF; the size of the printed electrode layer was $20 \mathrm{~mm} \times 40 \mathrm{~mm}$ with an activated layer that was $65 \mu \mathrm{m}$ thick. The same electrodes were used for the both the positive and the negative electrodes in our EDLC design. Then, we place a line of UV curable resin (World Rock, Kyo-ritsu chemical \& Co. Ltd.) around the electrode layer using an automated dispenser (Desktop Robot Shotmaster 300, Musashi Engineering, Inc.), and curing by UV irradiation (Spot light source LC8 Lighting Cure: L9566, Hamamatsu photonics K.K.), thereby forming a frame around the carbon electrode layer on the positive electrode. Only the positive electrode has a rectangular frame around the electrode layer, it being $300 \mu \mathrm{m}$ in height and 1000 $\mu \mathrm{m}$ line in width. On the other hand, all of the aluminum oxide surface on the negative electrode was covered by polyphenylenesulfide tape in order to prevent contact between the positive and the negative current collectors.

The PSiP/IL solid electrolyte (PSiP / DEME-TFSI = 75 / 25 (wt.\%)) layer was formed by a casting method on the dried positive activated carbon (AC) electrode. We prepared a mixed solution, composed of PSiP, the ionic liquid DEME-TFSI and propylene carbonate $(41.25: 13.75: 45.00$; weight ratio) to pour into the frame on the positive electrode surface, keeping the whole under reduced pressure at 25 ${ }^{\circ} \mathrm{C}$ for $3 \mathrm{hrs}$ in order to allow the liquid to penetrate the electrode. And then, we leftt 
the electrode at $50{ }^{\circ} \mathrm{C}$ under reduced pressure for 3 days to evaporate the organic solvent, until its weight became constant. The thickness of the solid film thus prepared was about $200 \mu \mathrm{m}$. A bipolar electrode was constructed by welding/connecting the negative and positive electrodes using the uncoated back sides of the aluminum oxide foil. Two sheets of bipolar electrode that had PSiP/IL solid electrolyte on the positive side were sandwiched between single-side coated positive and negative electrodes such that the positive and negative electrodes faced each other. Just before assembly, the activated carbon layer of the negative electrode was wet with a small amount of DEME-TFSI. Finally, the assembled cell body was inserted into a bag made of an aluminum-laminated thermoplastic film, pressed, annealed at $80{ }^{\circ} \mathrm{C}$ and sealed in it by hot pressing at $190{ }^{\circ} \mathrm{C}$ under reduced pressure. All of these fabrication processes were carried out in a dry atmosphere, with the dew point of air less than $-40{ }^{\circ} \mathrm{C}$. The prepared cell had a capacitance of about $0.41 \mathrm{~F}$, and the nominal operating voltage of the cell ranged from $0 \mathrm{~V}$ to 7.5 V.

We also prepared normal EDLC cells that had a pair of single-side coated electrodes (operation voltage; $2.5 \mathrm{~V}$ ) with a PSiP/IL solid electrolyte, non diluted DEME-TFSI (neat ionic liquid) and 1M DEME-TFSI in propylene carbonate as an electrolyte, respectively. For the liquid electrolyte cell, paper (40 $\mu \mathrm{m}$ of thickness) made from cellulose fiber was used as a separator. These normal EDLC cells had a capacitance of about $1.25 \mathrm{~F}$. 
<Fig. 2>

\subsection{Charge / discharge operation and other measurements}

Prepared EDLC cells were charged in a constant current (CC) mode from $V=0$ to $7.5 \mathrm{~V}$ at $I=0.93 \mathrm{~mA}$, corresponding to $1.0 \mathrm{C}$. At the full voltage, the cell was then further charged to at a constant voltage $(7.5 \mathrm{~V})$ for $15 \mathrm{~min}$ (i.e. $\mathrm{CV}$ mode). It was then discharged to $0 \mathrm{~V}$ at a given temperature $T$ in $\mathrm{CC}$ mode at currents that varied from $I=0.93$ to $465.0 \mathrm{~mA}$. The corresponding energy output $W$ was calculated from the discharge curve via the relation $W=\int I V \mathrm{~d} t$ [5]. The capacitance per unit weight was found by dividing the capacitance by the total weight of the active carbon material in a pair of electrodes. The cycle stability within the initial 1000 cycles of an EDLC was confirmed by charging at $7.5 \mathrm{~V}$ or $2.5 \mathrm{~V}$ (in the case of the conventional EDLC cell) and discharging to $0 \mathrm{~V}$. The cycle test was conducted by repeating 99 cycles of 10C charge / discharge and a one-time $1 \mathrm{C}$ charge / discharge process (once every 100 cycles) at $25{ }^{\circ} \mathrm{C}$. The deterioration of the capacity of the cell was measured at set intervals. All data reported here are the average of that from three similar cells. For the EDLC testing, we used a HJ100ISD8 charge-discharging system from HOKUTO DENKO Co. Ltd.

AC-impedance measurements of the solid electrolyte used a multi-frequency LCR meter (Agilent Technology E4980A Precision LCR meter). In order to evaluate the mobility of the ion, the self-diffusion coefficient $D$ of $\mathrm{DEME}^{+}$and 
TFSI $^{-}$was eveluated by pulsed-gradient spin-echo NMR (PGSE-NMR) spectroscopy on a JEOL JEM-ECX400 spectrometer coupled with a pulse field gradient probe. Detailed measurement conditions are given in our previous paper [16]. Electrochemical impedance spectroscopic measurements of fully-charged cells were measured with an impedance analyzer (Princeton Applied Research VersaSTAT3 coupled with FRA) over a frequency range of $100 \mathrm{KHz}$ to $10 \mathrm{mHz}$ at 2.5 or $7.5 \mathrm{~V}$. Scanning electron microscopy analysis was performed on a JEOL JSM-6510.

\section{Results and Discussion}

When the compatibility between the brush and solvent is high, the PSiP should be highly dispersed in the solvent without aggregation. In the case of PSiP having an ionic liquid type polymer brush being suspended in the ionic liquid DEME-TFSI, a liquid dispersion solution formed with iridescence at a particular concentration, indicating the formation of a colloidal crystal [16]. We succeeded in solidifing this colloidal crystal from the mixture of PSiP, ionic liquid and a volatile organic solvent, such as propylene carbonate, by a simple casting method. It was revealed a certain compositions, namely a mixture of $75 \mathrm{wt} \% \mathrm{PSiP}$ and $25 \mathrm{wt} \% \mathrm{IL}$ produced a solid film with sufficient physical strength to maintain its film shape (Fig. 1). A scanning electron microscopy (SEM) image of the cross section of PSiP/IL solid film treated by $\mathrm{UV} / \mathrm{O}_{3}$-etching indicated that the PSiPs were uniformly arranged over a wide area, reaching a size of tens of micrometers and 
forming a crystal-like structure, as shown in Fig. 1. From detailed SEM observations, it was clearly shown that the PSiP/IL solid film has a face-centered cubic (fcc) crystal structure and the distance between the centers of the the nearest neighbor PSiPs was $\approx 148 \pm 3 \mathrm{~nm}[16]$. A three-dimensional continuous ion conductive path of a few tens of nanometers in width will form in the gaps between particles due to the three-dimensional ordered array of PSiP where the poly(DEMM-TFSI) brush is swollen with DEME-TFSI, giving a high conductivity.

The PSiP/IL solid electrolyte had a high ionic conductivity, with a $\sigma$ value of $0.13 \mathrm{mS} \mathrm{cm}^{-1}$ at $25^{\circ} \mathrm{C}$. Despite the 64 weight $\%$ of non-conductive silica particles, the conductivity was very high, more than three orders of magnitudes greater than that of the bulk brush component polymer solid film, poly(DEMM-TFSI) $(\mathrm{Mn}=25,000)$. Further, the PSiP/IL solid electrolyte film showed thermoplasticity above $80{ }^{\circ} \mathrm{C}$, enabling intimate contact between the counter-electrodes and electrolyte surface by hot pressing.

Since the PSiP solid had strength sufficient to prevent contact of the electrodes and good liquid retention properties, we were able to design and fabricate a demonstration bipolar EDLC cell. In the bipolar design, since multiple unit cells composed of pairs of negative and positive electrodes are connected in series in one cell package, it is possible to not only obtain high voltages, but it is also possible to minimize the ohmic loss between adjacent unit cells. As a result, uniform current and voltage distributions over the electrode surface is achieved 
and high rates can be obtained compared to monopolar devices. Applying the bipolar design to a high voltage power supply, can reduce the total cost by simplification of the control circuit and weight savings. However, leakage from a solidified electrolyte could cause a short circuit between the series connected unit cells, which would probably lead to a fatal outcome. Accordingly, for highly reliable bipolar EDLC cells, the solid electrolyte not only needs to exhibht high ionic conductivity, but shoud also have good liquid retention properties over a wide temperature range. There have been no reports of the development of such a reliable bipolar EDLC using a traditional gel electrolyte.

Fig 3. shows the charge and discharge curves of the experimental 7.5V EDLC cell. As intended, the charging and discharging operations were successfully performed between 0 and $7.5 \mathrm{~V}$, a value three times as high as the unit-cell voltage. The EDLC has shown a relatively small ohmic resistance (IR drop) and standard capacitive behavior, such as a quite linear slope for both charge and discharge processes. In the case of the $7.5 \mathrm{~V}$ EDLC cell, the capacitance was $0.41 \mathrm{~F} /$ cell at $1 \mathrm{C}$ current $(0.93 \mathrm{~mA})$ discharge, corresponding to about $21 \mathrm{~F} / \mathrm{g}$ of specific capacity per activated carbon weight, and the Coulombic efficiency was $99.15 \%$ at 100 cycles.

<Fig. 3>

The discharge rate performance of the EDLC cells $(7.5 \mathrm{~V}$ and $2.5 \mathrm{~V})$ using a 
PSiP/IL solid electrolyte was measured and compared with the case of the cells with liquid-type electrolytes, such as the non-diluted, neat ionic liquid and a $1 \mathrm{M}$ concentration of ionic liquid in propylene carbonate solution at $25^{\circ} \mathrm{C}$. A high rate of discharge is one of the features of EDLCs, however the relative capacity (\%), that is the retention ratio of the capacity after a $1 \mathrm{C}$ discharge, decreased with an increase in the discharge rate. As shown in Fig. 4, the relative capacity up to a 10C (current density; $1.16 \mathrm{~mA} \mathrm{~cm}{ }^{-2}$ ) discharge showed almost the same trend, regardless of the kind of electrolyte. The newly-designed 7.5V-EDLC cell produced the equivalent output performance of a the conventional $2.5 \mathrm{~V}$-EDLC with organic liquid electrolyte in the relatively low output current range. However, in the high power discharging region of more than a 50C rate, the relative capacity of the EDLCs using the neat IL and the PSiP/IL solid electrolyte decreased much faster than that of a device using organic liquid electrolyte as the discharge current increased. These properties are probably due to the high impedance of the PSiP/IL (7.5V) solid electrolyte cell, measured as $13.37 \Omega$ at $1 \mathrm{KHz}$, compared to $1.56 \Omega$ for the neat IL $(2.5 \mathrm{~V})$, and $3.99 \Omega$ for the PSiP/IL solid (2.5V) cells, respectively, while the organic liquid electrolyte cell was $0.77 \Omega$.

However, because the 7.5V EDLC cell not only had about 70\% relative capacity at $100 \mathrm{C}$ discharge (current density; $11.63 \mathrm{~mA} \mathrm{~cm}^{-2}$ ), but also can withstand liquid leakage and has flame resistant properties, it seemed that this cell had features that would make it a practical choice as an electric component. The 7.5V EDLC using a PSiP/IL solid electrolyte with a prismatic type aluminum laminate packaged cell 
(Fig. 2) has a power density of $185 \mathrm{~W} / \mathrm{kg}$ and $204 \mathrm{~W} / \mathrm{L}$ during 50C discharging at the nominal voltage [17].

$<$ Fig. 4>

Nyquist plots, over a measurement frequency range from $10 \mathrm{mHz}$ to $100 \mathrm{KHz}$ of the demonstration cells are shown in Figure 5. They consist of three parts, a semicircle at the high frequency field, followed by an inclined line and a vertical line in the low frequency region.

The intersection point with the real axis at high frequency that is the location of the lowest resistance value on the real axis indicates the resistance of a cell that generally originates from the bulk resistance of the electrolyte, separator and external circuit resistance $\left(R_{s}\right)$. The broadness of the semi-circle that is the chord between the high frequency intercept $\left(R_{S}\right)$ and the low frequency intercept, corresponds to the charge transfer resistance $\left(R_{c t}\right)$. The straight line with a slope of $45^{\circ}$ is related to the Warburg impedance $(W)$ created by the diffusion of electrolyte ions. The vertical line to the real axis is caused by purely capacitive behavior $[18,19]$. An equivalent circuit for the EDLC cells in shown in the inset of Fig. 5. In the four tested systems, $R_{S}$ is attributed to the resistance of electrolytes. The order of the $R_{s}$ values of each cell is the same as the order of ionic conductivity of the each electrolyte. The $R_{s}$ was $0.34,0.95,3.52$ and $12.15 \Omega$ for conventional organic liquid $(2.5 \mathrm{~V})$, the neat IL $(2.5 \mathrm{~V})$, the PSiP/IL solid $(2.5 \mathrm{~V})$ and the PSiP/IL solid 
(7.5V) electrolyte cells, respectively, based on the equivalent circuit simulation. That of the $7.5 \mathrm{~V}$ cell with a PSiP/IL solid electrolyte is almost three times larger than that of the $2.5 \mathrm{~V}$ cell. If it is possible to reduce the thickness of the electrolyte film to $1 / 10$ of its present thickness (to $20 \mu \mathrm{m}$ ) by some improvement of the film forming process, the $R_{s}$ of the $7.5 \mathrm{~V}$ cell will be reduced to the same value as the cell using an ionic liquid electrolyte.

It is interesting that the semicircle in the high frequency region of the PSiP/IL cells that is associated with the interfacial resistance $R_{c t}$ between the electrode and electrolyte was remarkably small compared with that of the liquid electrolyte cells. The $R_{c t}$ was $1.97,1.99,0.62$ and $1.53 \Omega$ for the conventional organic liquid (2.5V), the neat IL $(2.5 \mathrm{~V})$, the PSiP/IL solid electrolyte $(2.5 \mathrm{~V})$ and $7.5 \mathrm{~V}$ cell, respectively. A small $R_{c t}$ may be a major feature of this solid electrolyte system. At medium frequencies, especially in the highly viscous liquid and solid electrolyte cells, a straight line with a slope of $45^{\circ}$ appears and this impedance reflects the ion diffusion phenomena and subsequently, all the cells behave like a pure EDLC capacitor which is characterized by a vertical line at low frequencies. For the cell with IL and PSiP/IL solid electrolyte, the output characteristics showing a high current value deteriorated (Fig. 4), due to the large diffusion resistance and higher total resistance.

$<$ Fig. 5> 
It has generally been believed that the solidification of the electrolyte greatly inhibits ion mobility, and thereby the ionic conductivity would be severely reduced. For example, the ionic conductivity of the polymer material poly(DEMM-TFSI) $(M n=25,000)$ that was obtained by polymerization of the polymerizable ionic liquid (DEMM-TFSI) is four orders of magnitude or more lower than that of the monomer. We measured the self-diffusion coefficient $D$ of $\mathrm{DEME}^{+}$and $\mathrm{TFSI}^{-}$(detected as ${ }^{1} \mathrm{H}$ and ${ }^{19} \mathrm{~F}$, respectively) using pulsed-gradient spin echo NMR (GSE-NMR) spectroscopy [16] for comparison with the ionic mobility in the PSiP/IL solid, neat IL electrolyte and ionic liquid type polymer solution to match the polymer concentration of the polymer brush portion in PSiP/IL electrolyte.

The results are summarized in Table $\mathrm{S} 1$ in the supporting information. The $D$ value of both the cation and anion in the PSiP/IL solid electrolyte showed a very high value that was about half of the $D$ value in the ionic liquid, despite the measurement being in the solid. Compared to a polymer solution, this value was about twice as large as the $D$ value of the solution having the same composition (poly(DEMM-TFSI): IL= 72:28) as the ion conducting path in the PSiP/IL solid electrolyte. The concentrated polymer brush might have a positive effect in enhancing of the mobility of ions, though the detailed mechanism is not yet clear.

The leakage currents of EDLCs charged at $0.93 \mathrm{~mA}$ (current density; $0.12 \mathrm{~mA}$ $\mathrm{cm}^{-2}$ ) were measured at $25{ }^{\circ} \mathrm{C}$. The conventional $2.5 \mathrm{~V}$ cells were continuously charged at full operating voltage (CV-mode), and the leakage current was 
measured for 24 hrs, as shown in Fig. 6. The leakage current decreased with time. The PSiP/IL solid and neat IL electrolyte cell had leakage currents that stabilized faster than the organic liquid cell. It can be concluded that electrochemical decomposition was not occurring during the floating charge process in the solid electrolyte cell.

<Fig. 6>

The deterioration of the retention ratio of the cell capacity with full charge/discharge cycle number is indicated in Fig. 7. The cycle-durability evaluation is conducted by charge-discharge cycling in a galvanostatic mode at a constant current of $10 \mathrm{C}$ (i.e., $1.16 \mathrm{~mA} \mathrm{~cm} \mathrm{~cm}^{-2}$ ) 99 times, and the capacity was checked at $1 \mathrm{C}$ and 100 time intervals. With an increase in cycle number, the retention ratio of the capacity of the high voltage EDLC cell hardly changed, and showed good cycle stability. The retention ratio of capacity at the 1000 cycle mark was 96.2, 95.5, 95.6 and 94.7\% for the 7.5V, 2.5V PSiP/IL solid, 2.5V IL and the $2.5 \mathrm{~V}$ organic liquid electrolyte cells, respectively. The Coulombic efficiency of the PSiP/IL solid electrolyte $7.5 \mathrm{~V}$ cell at discharge after 1000 cycles was $99.7 \%$ at $10 \mathrm{C}$ discharge.

<Fig. 7> 
A spontaneous voltage drop of EDLCs when the cell is maintained without a load, is generally called 'self-discharge' [20,21]. The voltage decrease is just a storage energy loss of devices. This phenomenon is also seen in batteries, though it is more remarkable in EDLCs than in batteries. The degree of spontaneous voltage drop increased with increasing cell voltage and the storage temperature in general. The self-discharge properties of various EDLCs at $60{ }^{\circ} \mathrm{C}$ is shown in Fig. 8. The voltage drop of the PSiP/IL solid cell was found to be much smaller than that of the organic liquid electrolyte at a relatively high temperature of $60{ }^{\circ} \mathrm{C}$.

<Fig. 8>

\section{Conclusion}

We have developed a bipolar EDLC device with a high 7.5 V operating voltage using a newly-developed solid electrolyte (PSiP/IL solid electrolyte) in which silica particles decorated with an ionic liquid type polymer brush are regularly arranged in three-dimensions in the presence of a small amount of ionic liquid (IL). This leads to the existance of a continuous ion conductive pathway consists of IL and brush. The PSiP/IL solid electrolyte has extremely high ionic conductivity, one of the highest among solid electrolytes. An EDLC using a PSiP/IL solid electrolyte has high liquid retention, flame retardant properties, and highly cycle durability as good as conventional EDLC cells. It showed similar rate properties to 
a common cell using a flammable organic liquid electrolyte with a low operating voltage up to a $10 \mathrm{C}$ discharge. It is a special feature of this type of solid system capacitor that the leakage current during continuous charging at a constant voltage (floating charge) and the self-discharge properties, even at a relatively high temperature of $60{ }^{\circ} \mathrm{C}$, were extremely small compared to an organic liquid system. It is also evident that, by using a PSiP/IL solid electrolyte having a high liquid retention capacity, a gasket for preventing a short circuit by the liquid electrolyte was not required, so it is possible to design a simple and lightweight EDLC cell with a high operating voltage. It might well open a new route to developing a safe next generation of electrochemical devices.

\section{Acknowledgements}

The authors thank Mrs. Tadayoshi Akao and Zenzo Hashimoto, CorePro R\&D, Inc. for their cooperation in the design of a bipolar EDLC for an industrial producing process and valuable comments. A part of this work was supported by a Grant-in Aid for Scientific Research (No. 25630311 and 22550203) from the Ministry of Education, Science, Sport, and Culture, Japan, and by Grants for Core Research for Evolutional Science and Technology (CREST) from the Japan Science and Technology Agency. This work was supported by the Collaborative Research Program of Institute for Chemical Research, Kyoto University (grant No. 2011-29). 


\section{Dedication}

This research paper is dedicated to our late coworker Mr. Zenzo Hashimoto, President of CorePro R\&D, Inc.

\section{References}

[1] T. Morimoto, K. Hiratsuka, Y. Sanada, K. Kurihara, S. Ohkubo and Y. Kimura, Proc. 33rd Int. Power Sources Symposium. Cherry Hills. USA, 1988. p. 618.

[2] T. Morimoto, K. Hiratsuka, Y. Sanada, K. Kurihara, Electric double-layer capacitor using organic electrolyte, J. Power Sources 60 (2) (1996) 239-247.

[3] K. Yuyama, G. Masuda, H. Yoshida, T. Sato, Ionic liquids containing the tetrafluoroborate anion have the best performance and stability for electric double layer capacitor applications, J. Power Sources 162 (2006) 1401-1408.

[4] Andrew Burke, Marshall Miller, Testing of electrochemical capacitors: Capacitance, resistance, energy density, and power capability, Electrochimica Acta 55 (2010) 7538-7548.

[5] T. Sato, G. Masuda, K. Takagi, Electrochemical properties of novel ionic liquids for electric double layer capacitor applications, Electrochimica Acta. 
49 (21) (2004) 3603-3611.

[6] Andrzej Lewandowski, Angelika Olejniczak, N-Methyl-N-propylpiperidinium bis(trifluoromethanesulphonyl)imide as an electrolyte for carbon-based double-layer capacitors, J. Power Sources 172 (1) (2007) 487-492.

[7] T. Sato, S. Marukane, T. Morinaga, Ionic Liquids for the Electric Double Layer Capacitor Applications, Intec CPT-6, Rijeka, Croatia, 2011.

[8] R. D. Rogers, K. R. Seddon, Ionic Liquids: Industrial Applications for Green Chemistry (Acs Symposium Series), American Chemical Society (2001) 0-84123-789-1.

[9] Hal-Bon Gu, Jong-Uk Kim, Hee-Woong Song, Gye-Choon Park, Bok-Kee Park, Electrochemical properties of carbon composite electrode with polymer electrolyte for electric double-layer capacitor, Electrochimica Acta.45 (2000) 1533-1536.

[10] Chun-Chen Yang, Sung-Ting Hsu, Wen-Chen Chien, All solid-state electric double-layer capacitors based on alkaline polyvinyl alcohol polymer electrolytes, Journal of Power Sources 152 (2005) 303-310.

[11] Shinji Nohara, Hajime Wada, Naoji Furukawa, Hiroshi Inoue, Masayuki Morita, Chiaki Iwakura, Electrochemical characterization of new electric double layer capacitor with polymer hydrogel electrolyte, Electrochimica Acta 48 (2003) 749-753.

[12] Chien-Ping Tien, Wuu-Jyh Liang, Ping-Lin Kuo, Hsi-Sheng Teng, Electric 
double layer capacitors with gelled polymer electrolytes based on poly(ethylene oxide) cured with poly(propylene oxide) diamines, Electrochimica Acta 53 (2008) 4505-4511.

[13] Shigeaki Yamazaki, Akihiko Takegawa, Yoshiro Kaneko, Jun-ichi Kadokawa, Masaki Yamagata, Masashi Ishikawa, An acidic cellulose-chitin hybrid gel as novel electrolyte for an electric double layer capacitor, Electrochemistry Communications 11 (2009) 68-70.

[14] Takaya Sato, Shoko Marukane, Takuya Narutomi, Tadayoshi Akao, High rate performance of a lithium polymer battery using a novel ionic liquid polymer composite, Journal of Power Sources 164 (2007) 390-396.

[15] Kazunari Soeda, Masaki Yamagata, Masashi Ishikawa, Outstanding features of alginate-based gel electrolyte with ionic liquid for electric double layer capacitors, Journal of Power Sources 280 (2015) 565-572.

[16] Takaya Sato, Takashi Morinaga, Shoko Marukane, Takuya Narutomi, Tatsuya Igarashi, Yuko Kawano, Kohji Ohno, Takeshi Fukuda, Yoshinobu Tsujii, Novel Solid-State Polymer Electrolyte of Colloidal Crystal Decorated with Ionic-Liquid Polymer Brush, Advanced Materials 23 (42) (2011) $4868-4872$.

[17] JIS D-1401 "Electrical characteristics test methods of electric double layer capacitors for use in hybrid electric vehicles" established 2009 by Japanese Industrial Standards Committee (2009).

[18] Xi-miao LIU, Rui ZHANG, Liang ZHAN, Dong-hui LONG, Wen-ming 
QIAO, Jun-he YANG, Li-cheng LING, Impedance of carbon aerogel/activated carbon composites as electrodes of electrochemical capacitors in aprotic electrolyte, New Carbon Materials 22 (2) (2007) $153-158$.

[19] Jinhee Kang, John Wen, Shesha H.Jayaram, Aiping Yu, Xiaohui Wang, Development of an equivalent circuit model for electrochemical double layer capacitors (EDLCs) with distinct electrolytes, Electrochimica Acta 115 (2014) 587-598.

[20] A.Lewandowski, P.Jakobczyk, M.Galinski, M.Biegun, Self-discharge of electrochemical double layer capacitors, Phys.Chem.Chem.Phys 15(22) (2013) 8692-9.

[21] B.W. Ricketts, C. Ton-That, Self-discharge of carbon-based supercapacitors with organic electrolytes, Journal of Power Sources 89 (1) (2000) 64-69. 


\section{Figure captions.}

Fig. 1. Preparation process for the novel PSiP/IL solid electrolyte.

Schematic illustration of a novel PSiP/IL-solid electrolyte with three-dimensional assembled PSiPs, thereby a forming network channel with high ion conductivity. Photograph of the solid film produced and the structure of the PSiP array in the solid analyzed from an SEM image of a fractured surface of the PSiP/IL-solid electrolyte observed at a magnification of $15,000 x$.

Fig. 2. Preparation process for a 7.5V EDLC cell with a bipolar design.

Fig. 3. Charge and discharge curves for the $7.5 \mathrm{~V}$ and $2.5 \mathrm{~V}$ EDLC cells with the PSiP/IL solid electrolyte at $25{ }^{\circ} \mathrm{C}$.

Fig. 4. Variation of the relative capacity with discharge current rate for EDLCs. The evaluated EDLCs were: (red filled circles, 9 ) the 7.5V-EDLC with PSiP/IL solid electrolyte; (blue filled circles, 0 ) the 2.5V-EDLC with PSiP/IL solid electrolyte; (black filled circles, ) the 2.5V-EDLC with neat ionic liquid, DEME-TFSI electrolyte; (open circles, $\bigcirc$ ) the 
2.5V-EDLC with organic liquid electrolyte, $1 \mathrm{M}$ DEME-TFSI in propylene carbonate. The charge was performed with $1 \mathrm{C}(0.93 \mathrm{~mA})$ to a predetermined voltage $(7.5 \mathrm{~V}$ or $2.5 \mathrm{~V})$ and the discharge with varying rates from $1 \mathrm{C}$ to $500 \mathrm{C}(465 \mathrm{~mA})$ to $0 \mathrm{~V}$ at $25{ }^{\circ} \mathrm{C}$. The $1 \mathrm{C}$ charge current was equivalent at a current density of $0.12 \mathrm{~mA} \mathrm{~cm}$. The horizontal axis indicates the charge and discharge cycle number and current density for (a) and (b), respectively, and vertical axis is the relative capacity for the 1C discharging capacity at 100 cycles for each cell.

Fig. 5. Experimental Nyquist plots of demonstration EDLCs with a frequency range from $100 \mathrm{KHz}$ to $10 \mathrm{mHz}$ at 2.5 or $7.5 \mathrm{~V}$ full charged state. The measurements were performed using a cell after 1000 charge-discharge cycles. The measured EDLCs were: (red filled circles, 0 ) the 7.5V-EDLC with PSiP/IL solid electrolyte; (blue filled circles, 0 ) the 2.5V-EDLC with PSiP/IL solid electrolyte; (black filled circles, 0 ) the 2.5V-EDLC with neat ionic liquid, DEME-TFSI electrolyte; (open circles, $\bigcirc$ ) the 2.5V-EDLC with organic liquid electrolyte, $1 \mathrm{M}$ DEME-TFSI in propylene carbonate. An equivalent circuit for the capacitor cells is shown in the inset of the figure.

Fig. 6. Variation of leakage current with time for demonstration EDLCs charged 
at $0.93 \mathrm{~mA}$ to a floating potential of $2.5 \mathrm{Vat} 25^{\circ} \mathrm{C}$. The measurements were performed using a cell after 1000 charge-discharge cycles. The measured EDLCs were: (blue filled circles, ) the 2.5V-EDLC with PSiP/IL solid electrolyte; (black filled circles, ) the 2.5V-EDLC with neat ionic liquid, DEME-TFSI electrolyte; (open circles, $\bigcirc$ ) the 2.5V-EDLC with organic liquid electrolyte, $1 \mathrm{M}$ DEME-TFSI in propylene carbonate.

Fig. 7. Cycle test of the demonstration EDLCs at $25{ }^{\circ} \mathrm{C}$. The measurements were performed using a cell after 1000 charge-discharge cycles. The measured EDLCs were: (red filled circles, ) the 7.5V-EDLC with PSiP/IL solid electrolyte; (blue filled circles, 0 ) the 2.5V-EDLC with PSiP/IL solid electrolyte; (black filled circles, $\mathrm{O}$ ) the 2.5V-EDLC with neat ionic liquid, DEME-TFSI electrolyte; (open circles, O) the 2.5V-EDLC with organic liquid electrolyte, 1M DEME-TFSI in propylene carbonate. The test cell was charged at constant current from $V=0$ to $2.5 \mathrm{~V}$ or $7.5 \mathrm{~V}$ at a constant current $I=1.16 \mathrm{~mA} \mathrm{~cm}^{-2}$ and rest $10 \mathrm{~min}$. Then the cell was discharged to $0 \mathrm{~V}$ at the same constant current. In order to measure the capacity retention ratio, the charging and discharging operation was similarly performed at the current of $I=0.12 \mathrm{~mA} \mathrm{~cm}^{-2}$ at the 100 times every. 
Fig. 8. Self-discharge of prepared EDLCs at $60^{\circ} \mathrm{C}$ after constant voltage charging. The charge was performed at $1 \mathrm{C}\left(0.93 \mathrm{~mA} \mathrm{~cm}^{-2}\right)$ to the predetermined full charge voltage $(7.5 \mathrm{~V}$ or $2.5 \mathrm{~V})$ and then charged at constant voltage for 15 min. The cells were kept standing in an oven at $60{ }^{\circ} \mathrm{C}$ for $48 \mathrm{hrs}$. The measurements were performed using a cell after 1000 charge-discharge cycles. The measured EDLCs were: (red filled circles, 9 ) the 7.5V-EDLC with PSiP/IL solid electrolyte; (blue filled circles, ) the 2.5V-EDLC with PSiP/IL solid electrolyte; (black filled circles, 0 ) the 2.5V-EDLC with neat ionic liquid, DEME-TFSI electrolyte; (open circles, $\bigcirc$ ) the 2.5V-EDLC with organic liquid electrolyte, $1 \mathrm{M}$ DEME-TFSI in propylene carbonate. 
Fig. 1.

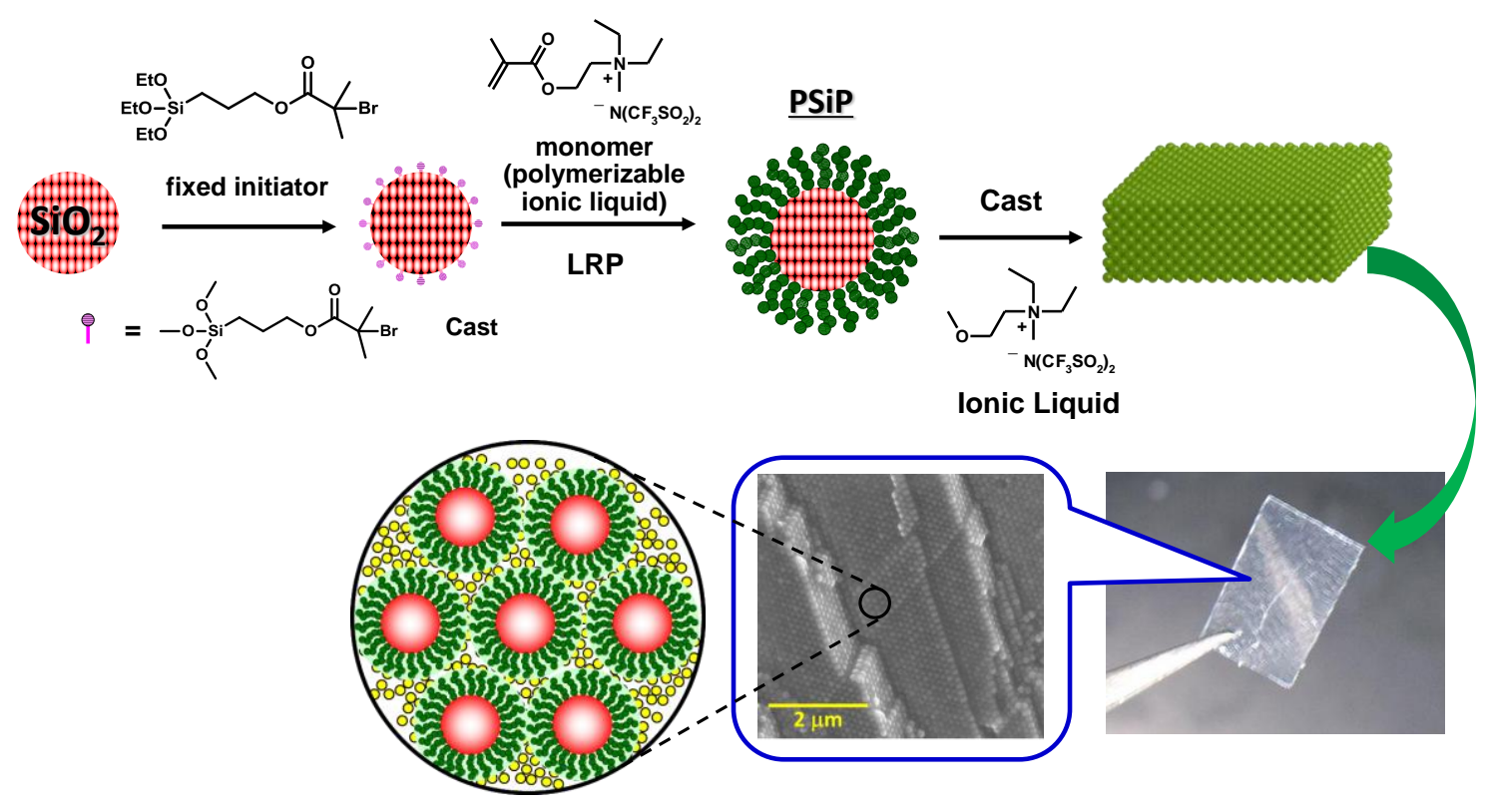


Fig. 2.

Positive Electrode Side

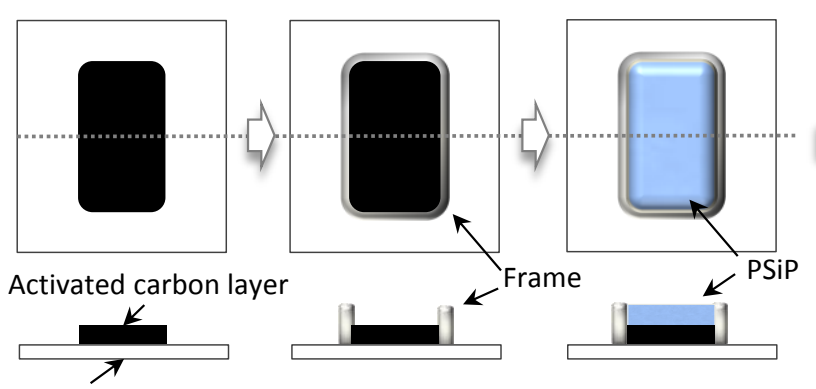

Aluminum oxide foil

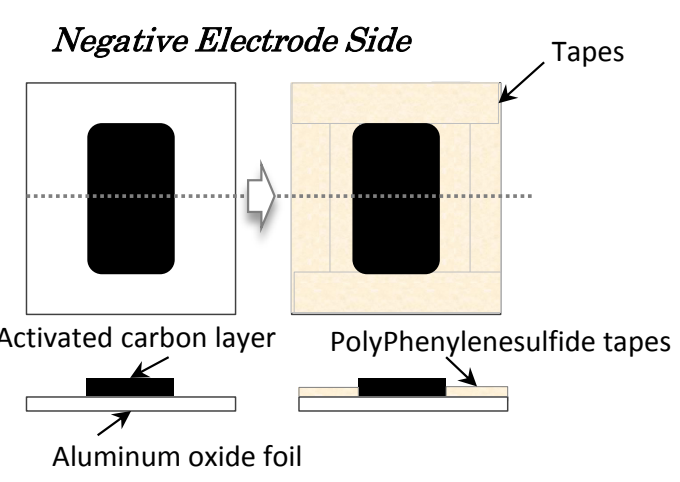

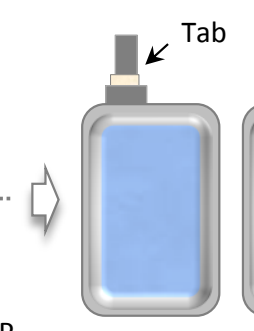

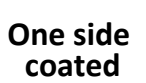

One side

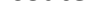

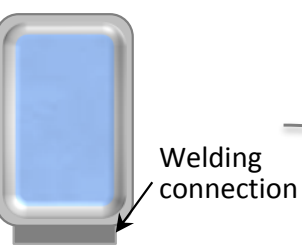

Bipolar electrode
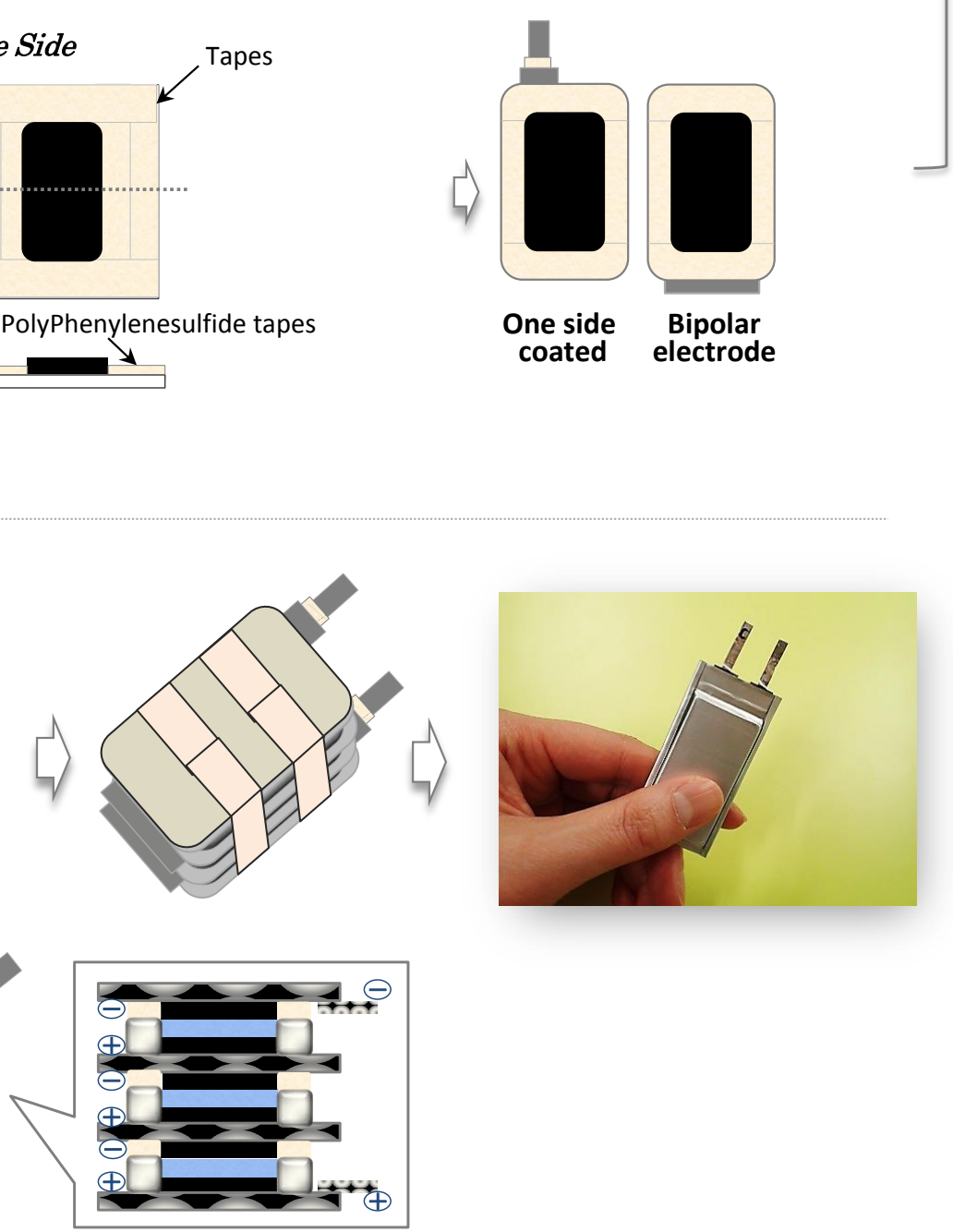
Fig. 3.

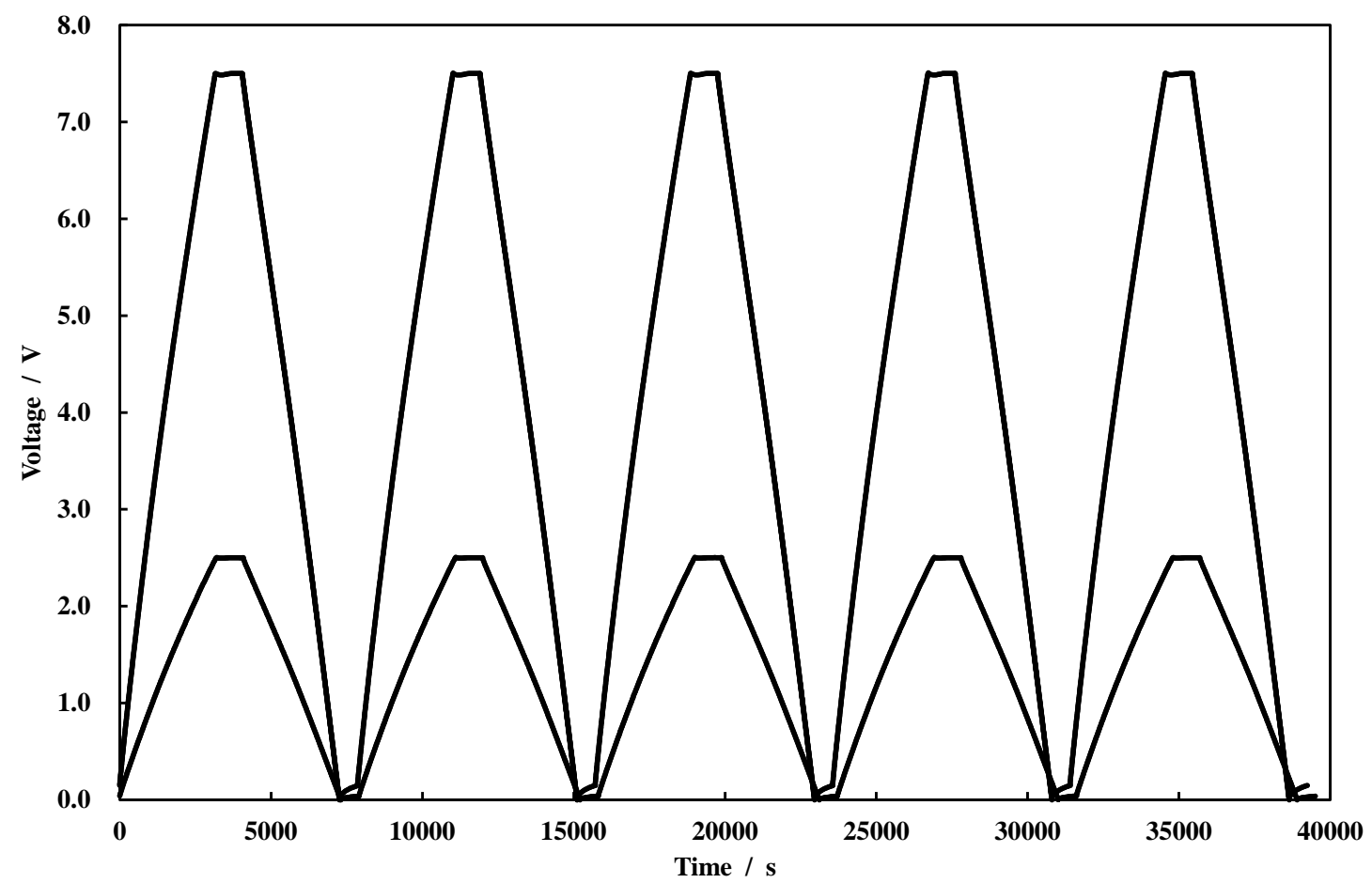


Fig. 4.
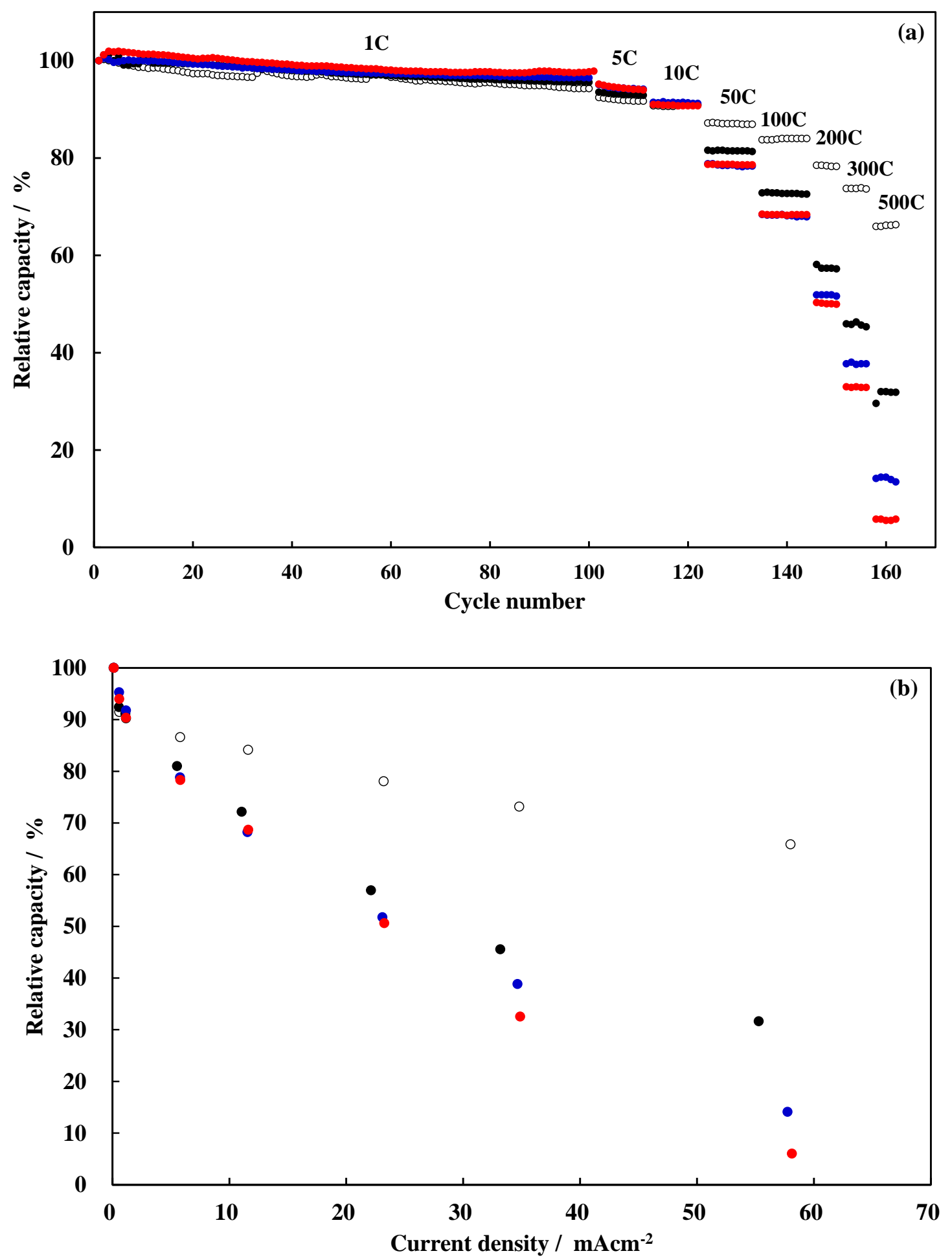
Fig.5

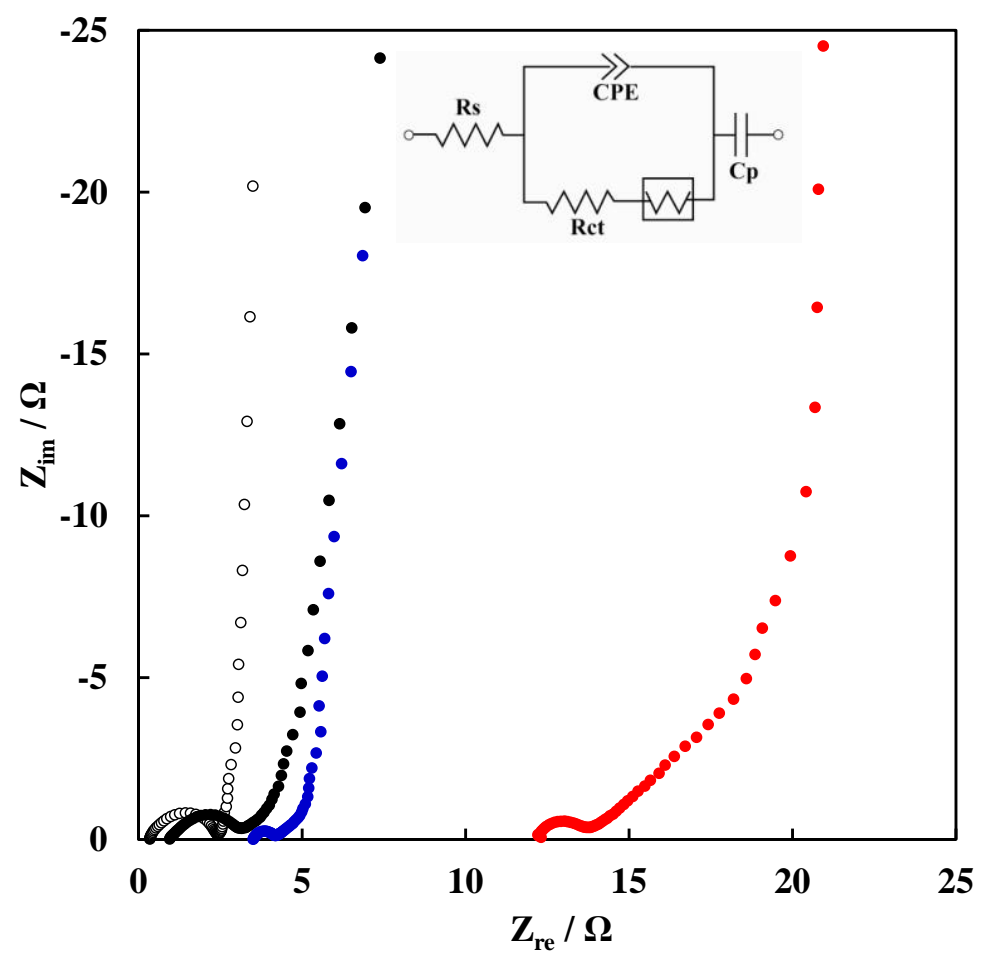


Fig.6

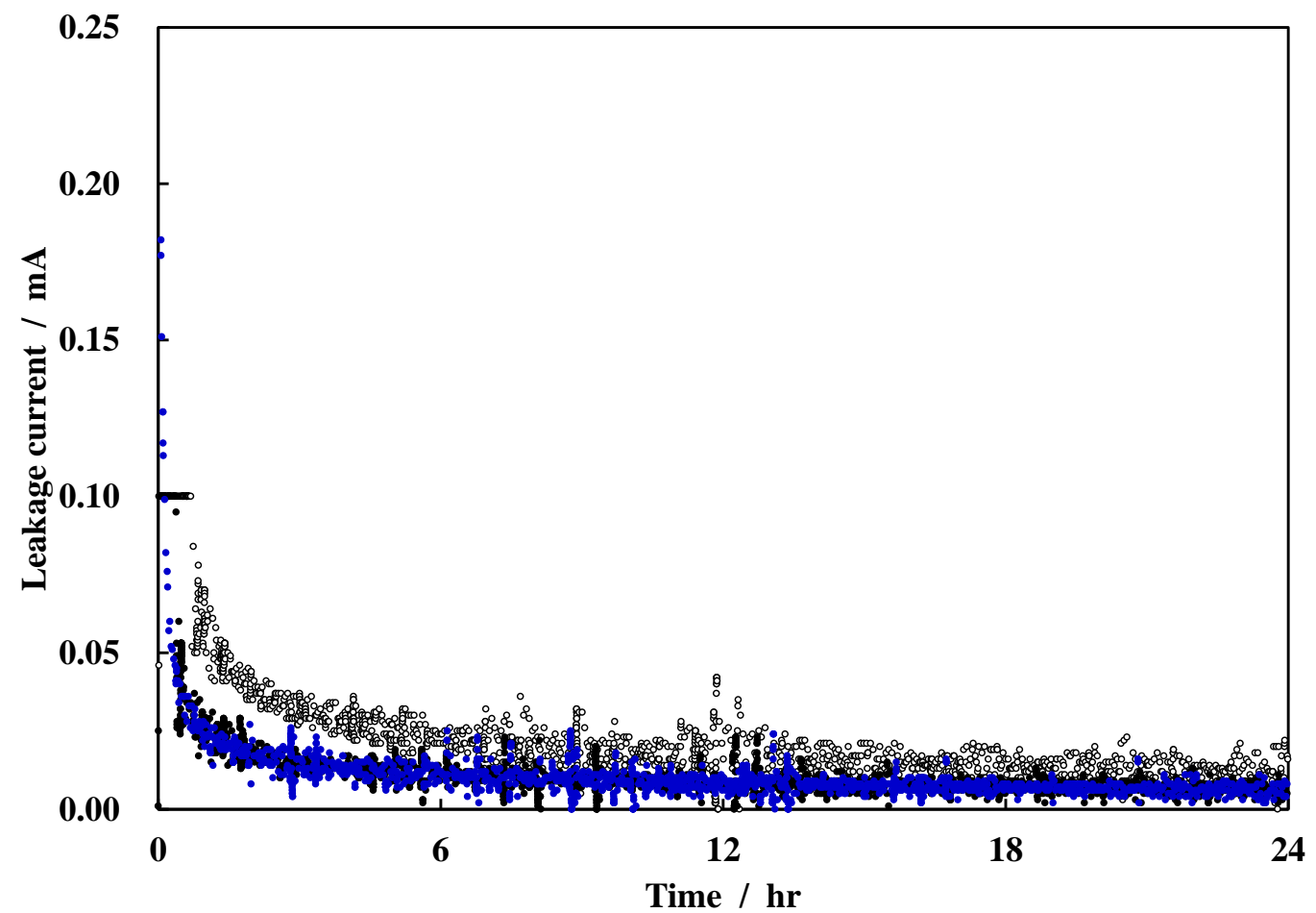


Fig.7

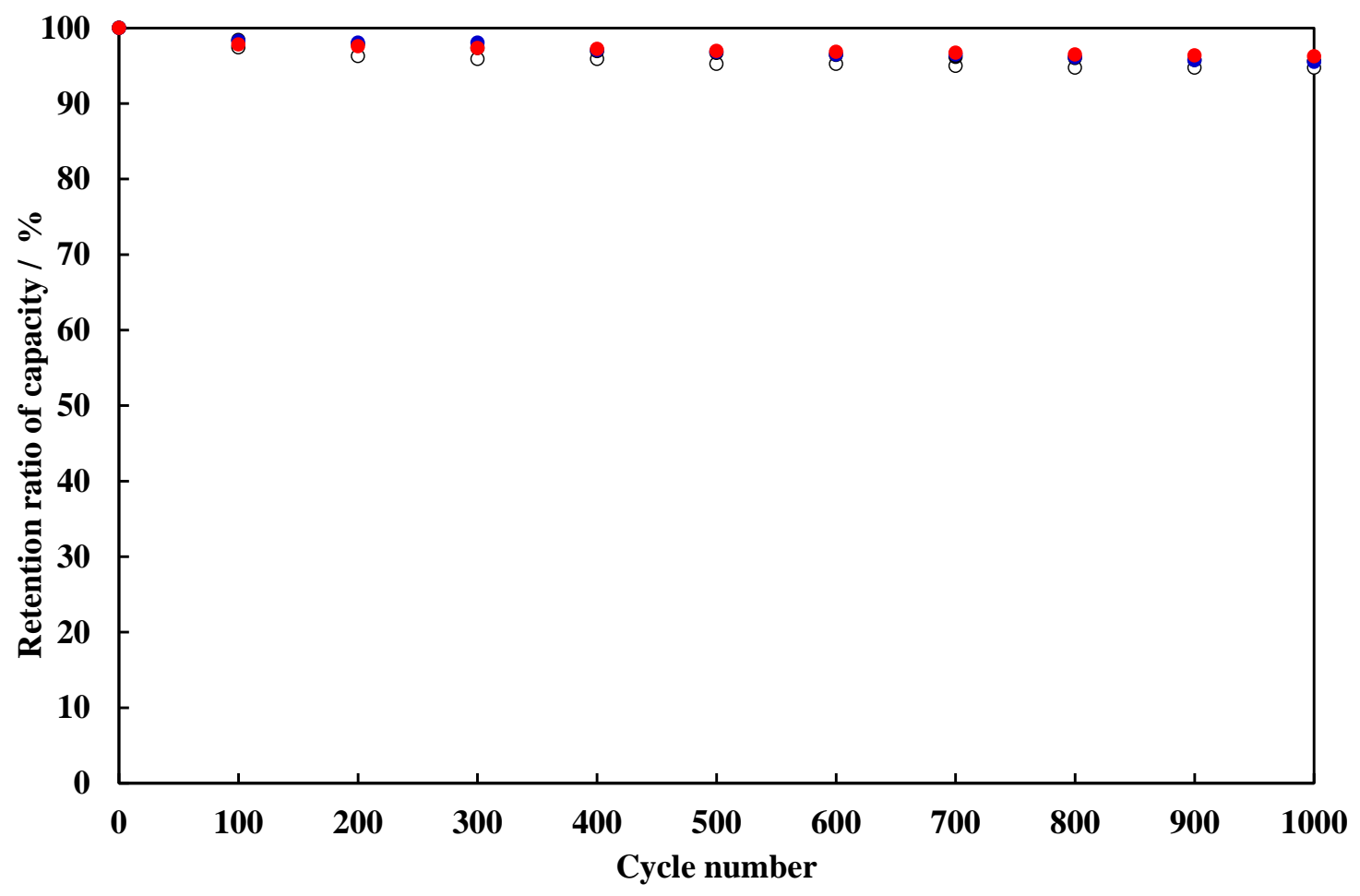


Fig.8.

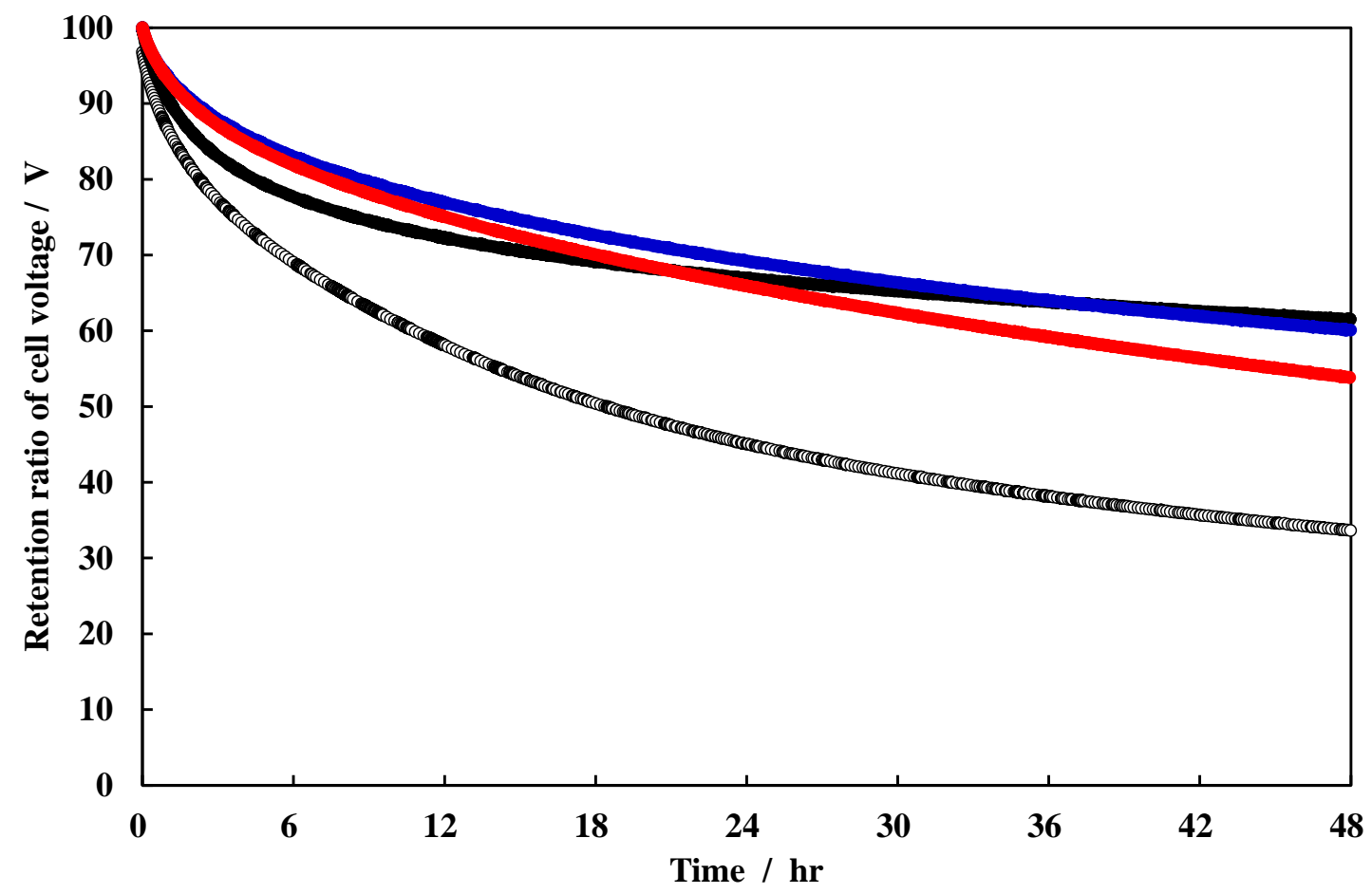


Graphical abstract

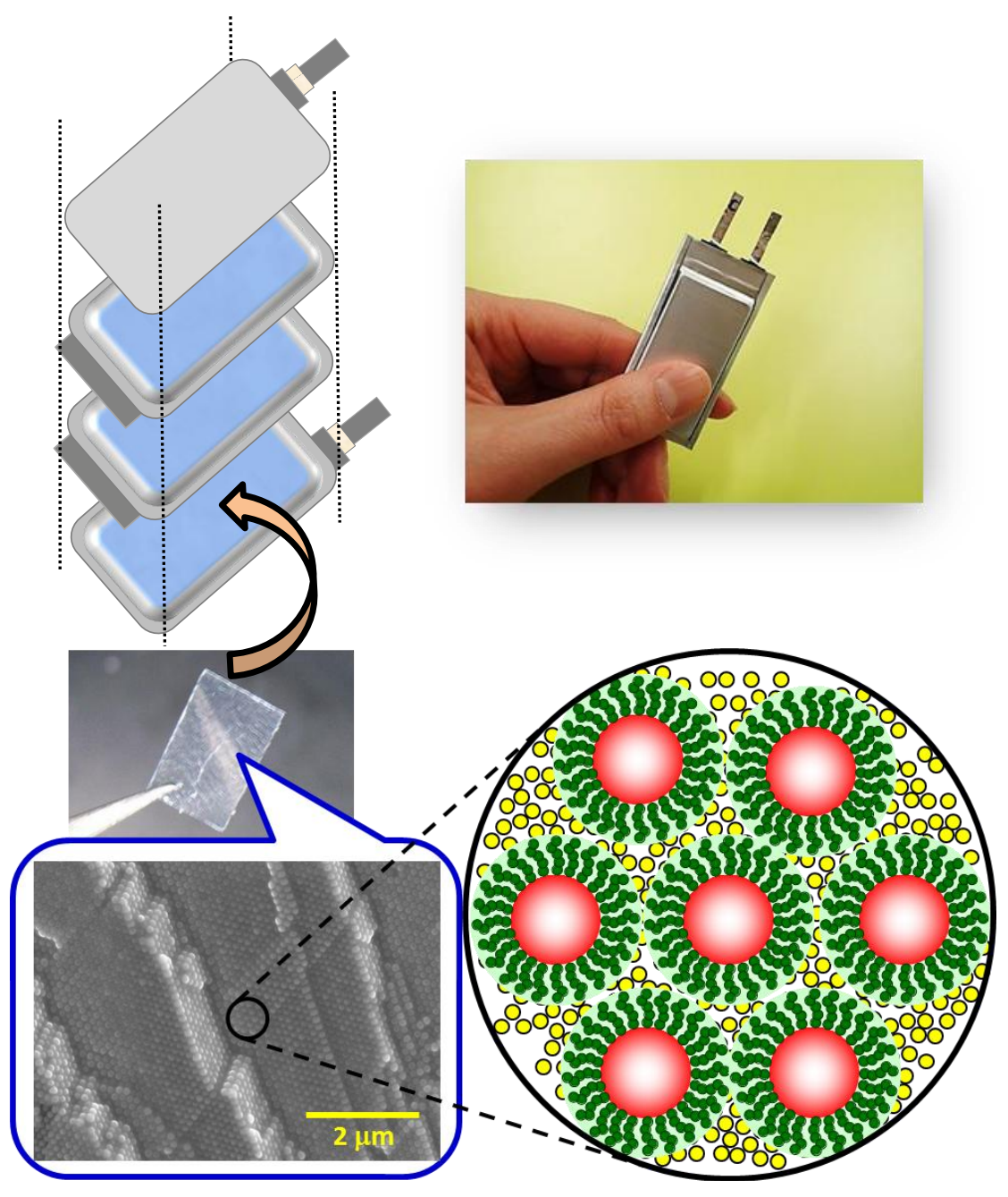

